\title{
Article \\ The Admissible Control Correction Method in a Nonlinear Terminal Perturbed Problem
}

\author{
Yuliya Belinskaya ${ }^{1,2}$, Mikhail Dmitriev ${ }^{1,3}$ (D) and Dmitry Makarov $1,3, *(\mathbb{D})$ \\ 1 Federal Research Center "Computer Science and Control" of Russian Academy of Sciences (FRC CSC RAS), \\ Pr. 60-Letiya Oktyabrya 9, 117312 Moscow, Russia; belinskaya.us@gmail.com (Y.B.); \\ mdmitriev@mail.ru (M.D.) \\ 2 Department of Mathematical Modelling, Bauman Moscow State Technical University (BMSTU), \\ 2nd Baumanskaya Str. 5, 105005 Moscow, Russia \\ 3 Department of Systems Research, Moscow Institute of Physics and Technology (MIPT), Institutskiy Per. 9, \\ 141701 Dolgoprudny, Russia \\ * Correspondence: makarov@isa.ru
}

check for updates

Citation: Belinskaya, Y.; Dmitriev, M.; Makarov, D. The Admissible Control Correction Method in a Nonlinear Terminal Perturbed Problem. Appl. Sci. 2021, 11, 5560. https://doi.org/10.3390/app11125560

Academic Editor: Jan Awrejcewicz

Received: 30 April 2021

Accepted: 7 June 2021

Published: 16 June 2021

Publisher's Note: MDPI stays neutral with regard to jurisdictional claims in published maps and institutional affiliations.

Copyright: (c) 2021 by the authors. Licensee MDPI, Basel, Switzerland. This article is an open access article distributed under the terms and conditions of the Creative Commons Attribution (CC BY) license (https:/ / creativecommons.org/licenses/by/ $4.0 /)$.

\begin{abstract}
A solution of a nonlinear perturbed unconstrained point-to-point control problem, in which the unperturbed system is differentially flat, is considered in the paper. An admissible open-loop control in it is constructed using the covering method. The main part of the obtained admissible control correction in the limit problem is found by expanding the perturbed problem solution in series by the perturbation parameter. The first term of the expansion is determined by A.N. Tikhonov's regularization of the Fredholm integral equation of the first kind. As shown by numerical experiments, the found structure of an admissible control allows one to find the final form of high precision point-to-point control based on the solution of an auxiliary variational problem in its neighborhood.
\end{abstract}

Keywords: nonlinear terminal control problem; perturbation method; flat system; covering method; Tikhonov's regularization; Fredholm integral equations

\section{Introduction}

Various approaches can be used to construct a point-to-point control, i.e., an admissible control that transfers a dynamic system to a given state in a finite time. One of them is associated with the use of algorithms for solving the introduced optimal control problems and application of the penalty function method to deal with the given terminal equality constraints. This method involves the use of an artificially introduced small parameter (an inverse value of the penalty coefficient). Under certain conditions, a solution of that problem leads in limit to an exact point-to-point control when the parameter tends to zero that means an unlimited increase of penalty coefficient. In that case, the introduction of a small parameter and the application of asymptotic expansions may lead to the construction of extrapolation procedures that make it possible to obtain open-loop and closed-loop controls with lower stiffness of calculations that translate the system into a given state with greater accuracy [1].

For nonlinear dynamical systems, approaches to solve problems with fixed ends are known only for some classes of systems. For example, for a statically linearizable system, the system can be transformed to a special form called the regular canonical form $[2,3]$, and then one can find the open-loop trajectory in the terms of time polynomials, the order of which is determined by the number of boundary conditions. For some dynamical systems, it is possible to transform the system to a quasi-canonical form [4], for which a similar approach is applied. A more general class of systems that includes statically linearizable systems are the so-called flat systems [5]. Each solution of a flat system is uniquely determined by a set of functions that are called the flat output of the system. For such systems, fairly general approaches to the open-loop trajectories design are also 
known. For example, a common approach is to construct linearizing feedback and time polynomial trajectory. Another promising algorithm that is applicable to both flat and non-flat systems is the covering method [6]. The method consists in expanding the phase space of the system by adding some subsystem (r-closure) and constructing a mapping (covering) from the extended phase space of the augmented system to the extended phase space of the original system. In this case, the solution of the two-point control problem is reduced to the solution of two related Cauchy problems. This method will be used in this work to find open-loop control for the unperturbed system. Among non-flat systems, we can point out Liouville systems [7], for which some approaches to solve two-point control problems are also known [8]. If the model contains perturbations in the form of an additional nonlinear function on the right-hand side with a small parameter, then a two-stage approach for searching the open-loop control can be proposed. At the first stage, the limiting unperturbed problem is solved. At the second stage, we take into account the asymptotics of the solution with respect to the perturbation parameter, so that a correction of the open-loop control for the unperturbed problem is constructed.

In this paper, we consider a solution in a nonlinear perturbed point-to-point control problem (or problem with fixed ends), where the limiting system is flat [5,9]. We construct the open-loop control for it using the covering method [6,10]. After that, it turns out that the control correction can be found with the help of the solution of regularized incorrectly posed problem obtained from first order asymptotics terms equations. Literature analysis showed that this approach was used for the first time.

\section{The Problem Statements}

Let us consider a perturbed nonlinear point-to-point control problem:

$$
\dot{x}=F_{0}(t, x, u)+\varepsilon F_{1}(t, x, u), \quad x\left(t_{0}\right)=x_{0}, \quad x\left(t_{f}\right)=x_{f}, \quad 0<\varepsilon \leq \varepsilon_{0} \ll 1 .
$$

Here, $\varepsilon_{0}$ is the upper bound on the values of the small parameter for which the proximate estimation of the asymptotic approximation takes place. For each specific problem, a specific value for the $\varepsilon_{0}$ parameter is defined, and in applications it can often be greater than 1.

The presence of the inequality $\varepsilon_{0} \ll 1$ in (1) only emphasizes that for small values of the parameter and under fairly general conditions on the right-hand sides, there are corresponding approximation estimates.

Let us denote an admissible trajectory and admissible a control in problem (1) by $x(t, \varepsilon) \in \mathbb{R}^{n}, u(t, \varepsilon) \in \mathbb{R}^{r}$. The small parameter $\varepsilon$ is considered known. We suppose that an admissible open-loop control $u_{0}(t)$ solving an unperturbed problem with fixed ends

$$
\dot{x}_{0}=F_{0}\left(t, x_{0}, u_{0}\right), \quad x_{0}\left(t_{0}\right)=x_{0}^{0}, \quad x_{0}\left(t_{f}\right)=x_{0}^{f}
$$

is known. It is assumed that the system in (2) is flat and its solution can be found by the covering method, but the perturbed system (1) is not flat.

Problem 1. It is necessary to find such an open-loop control $u_{1}(t, \varepsilon)=u_{0}(t)+\varepsilon u_{1}(t)$, where $\varepsilon u_{1}(t)$ is the main part of the $u_{0}(t)$ control correction, which solves perturbed nonlinear control problem (1) more precisely than $u_{0}(t)$.

Experiments show that when solving optimal control problems with a small parameter by traditional methods, asymptotic approximations, which can contain only part of the qualitative information about the exact solution (see in [11], for example), are of great help. This means that even with the help of local procedures, solutions can be found that are close to the points of the global extremum. Therefore, we may state the next problem. 
Problem 2. It is necessary to find control correction $\varepsilon \omega(t)$, which solves the problem

$$
\left\|x_{f}-x\left(t, u_{1}(t, \varepsilon)+\varepsilon \omega(t)\right)\right\| \rightarrow \min _{\omega} .
$$

Knowing $u_{1}(t, \varepsilon)$ means that the first order asymptotic approximation to the admissible open-loop control is known. Finding $\omega(t)$ actually means finding the missing part of the exact value of the admissible point-to-point control, the main part of which $u_{1}(t, \varepsilon)$ has already been found.

\section{Covering Method}

Here, we represent the covering method used to find an open-loop control for the unperturbed system.

Let $\mathcal{X}$ and $\mathcal{Y}$ be two given dynamical systems. A surjective mapping $v$ of the extended phase space of the system $\mathcal{X}$ into the extended phase space of the system $\mathcal{Y}$ is called a covering of $\mathcal{X}$ into $\mathcal{Y}$ if the following conditions hold:

(i) the mapping $v$ is regular, i.e., at any point rank of differential $d v$ is equal to the dimension of the image;

(ii) the mapping $v$ maps each trajectory of the system $\mathcal{X}$ into a trajectory of the system $\mathcal{Y}$;

(iii) the mapping of trajectories is regular, i.e., at any point tangent vector to a trajectory does not belong to kernel of $d v$.

From the definition of covering it follows that the preimage of any trajectory of $\mathcal{Y}$ consists of points of trajectories of some subsystem of $\mathcal{X}$.

Let us consider the solution of the point-to-point problem using covering method.

Let system

$$
\dot{x}=f(t, x, u), \quad x \in \mathbb{R}^{n}, \quad u \in \mathbb{R}^{m},
$$

be given, $\mathcal{O}$ be a domain of the space with coordinates $x, u, \dot{u}, \ldots, u^{(k)}, S_{0} \subset \mathcal{O}$ an initial set, $S_{f} \subset \mathcal{O}$ a target set. For a pair of vector functions $x(t), u(t)$ and a number $t_{1}$ denote by $j_{k}\left(x\left(t_{1}\right), u\left(t_{1}\right)\right)$ the point $x\left(t_{1}\right), u\left(t_{1}\right), \dot{u}\left(t_{1}\right), \ldots, u^{(k)}\left(t_{1}\right)$ of this space.

Knowing the final time $t_{f} \in\left(t_{0}, \infty\right)$, the problem is to compute an admissible input signal $u:\left[t_{0}, t_{f}\right] \rightarrow \mathbb{R}^{m}$ such that the system trajectory satisfies

$$
\begin{aligned}
& \forall t \in\left[t_{0}, t_{f}\right] \quad j_{k}(x(t), u(t)) \in \mathcal{O} \\
& j\left(x_{k}\left(t_{0}\right), u\left(t_{0}\right)\right) \in S_{0}, \quad j_{k}\left(x\left(t_{f}\right), u\left(t_{f}\right)\right) \in S_{f} .
\end{aligned}
$$

Let $g_{1}: \mathcal{O} \rightarrow \mathbb{R}^{n_{1}}$ and $g_{2}: \mathcal{O} \rightarrow \mathbb{R}^{n_{2}}$ be two mappings. Consider the case when the initial set $S_{0}$ and the target set $S_{f}$ are determined by the equalities

$$
\begin{aligned}
& S_{0}: g_{1}\left(x, u, \dot{u}, \ldots, u^{(k)}\right)=g_{1,0}, \\
& S_{f}: g_{2}\left(x, u, \dot{u}, \ldots, u^{(k)}\right)=g_{2, f} .
\end{aligned}
$$

Suppose functions $U_{i}$ and $\phi_{j}, i=\overline{1, m}, j=\overline{1, n_{2}}$, of the variables

$$
\begin{aligned}
& x_{1}, \ldots, x_{n}, u_{1}, \dot{u}_{1}, \ldots, u_{1}^{\left(k_{1}-1\right)}, u_{2}, \ldots, u_{m}^{\left(k_{m}-1\right)}, \\
& \forall i \quad k_{i} \leq k, \quad k_{1}+\ldots+k_{m}+n=n_{1}+n_{2}
\end{aligned}
$$

satisfy the following conditions.

(A) The relations $p_{j}=\phi_{j}, j=\overline{1, n}$, define a covering from the system

$$
\begin{aligned}
\dot{x}_{j} & =f_{j}\left(t, x_{1}, \ldots, u_{m}\right), \quad j=\overline{1, n}, \\
u_{i}^{\left(k_{i}\right)} & =U_{i}\left(t, x_{1}, \ldots, u_{m}^{\left(k_{m}-1\right)}\right), \quad i=\overline{1, m},
\end{aligned}
$$


into a system of the form

$$
\dot{p}=P(t, p), \quad p \in \mathbb{R}^{n_{2}} .
$$

(B) The given final value $g_{2, f}$ uniquely determines the value $p_{f}=p\left(t_{f}\right)$. Conversely, the value $p\left(t_{f}\right)$ uniquely determines the value $g_{2, f}$.

(C) If $p(t)$ is the solution of system (9) satisfying the condition $p\left(t_{f}\right)=p_{f}$, then the nonlinear vector equation

$$
p\left(t_{0}\right)=\phi\left(t_{0}, x_{1,0}, \ldots, x_{1,0}, u_{1}\left(t_{0}\right), \ldots u_{m}^{\left(k_{m}-1\right)}\left(t_{0}\right)\right)
$$

is solvable for $u_{1}\left(t_{0}\right), \ldots, u_{m}^{\left(k_{m}-1\right)}\left(t_{0}\right)$.

If conditions (A), (B), and (C) hold, then the problem (3)-(6) can be solved as follows.

1. The value $p\left(t_{f}\right)$ is found from the final conditions (6).

2. One finds the solution $p(t)$ of system (9) satisfying the condition $p\left(t_{f}\right)=p_{f}$ (that is, the solution of the Cauchy problem in reverse time, from $t_{f}$ to $\left.t_{0}\right)$.

3. One computes $p_{0}=p\left(t_{0}\right)$.

4. The values $u_{1}\left(t_{0}\right), \dot{u}_{1}\left(t_{0}\right), \ldots, u_{m}^{\left(k_{m}-1\right)}\left(t_{0}\right)$ are found from system (10).

5. By solving the Cauchy problem for system (7) and (8) with the initial values

$$
t_{0}, x_{1,0}, \ldots, x_{n, 0}, u_{1}\left(t_{0}\right), \dot{u}_{1}\left(t_{0}\right), \ldots, u_{m}^{\left(k_{m}-1\right)}\left(t_{0}\right),
$$

one finds a solution $(x(t), u(t))$ of system (3).

The solution thus found is a solution of the problem (3)-(6), because the function $x(t)$ satisfies the initial conditions by construction and the final conditions by virtue of condition (B). Using the flatness of the system (3), one can find the open-loop control for the problem with fixed ends.

\section{The Control Correction}

Let us represent $x(t, \varepsilon), u(t, \varepsilon)$ in the form of $\varepsilon$ integer powers series and substitute them in the conditions of problem (1), then in addition to (2) in the first approximation we obtain the following control problem with fixed ends

$$
\begin{gathered}
\dot{x}_{1}=F_{0, x}\left(t, x_{0}(t), u_{0}(t)\right) x_{1}(t)+F_{0, u}\left(t, x_{0}(t), u_{0}(t)\right) u_{1}(t)+F_{1}\left(t, x_{0}(t), u_{0}(t)\right), \\
x_{1}\left(t_{0}\right)=0, \quad x_{1}\left(t_{f}\right)=0,
\end{gathered}
$$

where $F_{0, x}=\frac{\partial F_{0}}{\partial x}, F_{0, u}=\frac{\partial F_{0}}{\partial u}$. Here, the unperturbed system is assumed to be flat and the control $u_{0}(t)$ is found using the covering method. The first (main) part of the control correction $u_{1}(t, \varepsilon)$ will be sought in the form $u_{1}(t, \varepsilon)=\varepsilon u_{1}(t)$. The term $\omega(t)$ is intended to clarify the solution in the original perturbed problem. The definition of $u_{1}(t)$ leads to the solution of the Fredholm equation of the first kind.

Therefore, determining $x_{0}(t), u_{0}(t)$ from problem (2), let us find $u_{1}(t)$ from (12) now, assuming $\omega(t)=0$. We have

$$
0=x_{1}\left(t_{f}\right)=\Phi\left(t_{f}\right) \int_{t_{0}}^{t_{f}}\left(\Phi^{-1}(\tau)\left(F_{0, u}\left(\tau, x_{0}(\tau), u_{0}(\tau)\right) u_{1}(\tau)+F_{1}\left(\tau, x_{0}(\tau), u_{0}(\tau)\right)\right)\right) d \tau,
$$

where $\Phi(t)$ is a fundamental matrix for a linear system $\dot{y}=F_{0, x}\left(x_{0}(t), u_{0}(t)\right) y$, i.e., $\dot{\Phi}=F_{0, x}\left(x_{0}(t), u_{0}(t)\right) \Phi, \Phi\left(t_{0}\right)=I$. Given that $\Phi(t)$ is a non-degenerate matrix for any $t$, we obtain the next Fredholm matrix integral equation of the first kind

$$
\int_{t_{0}}^{t_{f}} K(\tau) u_{1}(\tau) d \tau=b
$$


where

$$
K(\tau)=\Phi^{-1}(\tau) F_{0, u}\left(\tau, x_{0}(\tau), u_{0}(\tau)\right), \quad b=-\int_{t_{0}}^{t_{f}}\left(\Phi^{-1}(\tau) F_{1}\left(\tau, x_{0}(\tau), u_{0}(\tau)\right)\right) d \tau .
$$

It is known that equations of this type are incorrectly posed that means small changes in the conditions of the problem caused by the approximate nature of solution of (13), that occur during computation may lead to non-uniqueness of the solution and significant changes in the obtained $u_{1}$ value. We use the Tikhonov's regularization [12] (see also in [13]), according to which the approximate solution in (13) is found as a vector minimizing the next functional

$$
\begin{gathered}
M^{\alpha}\left(u_{1}\right)=\left(\int_{t_{0}}^{t_{f}} K(\tau) u_{1}(\tau) d \tau-b\right)^{\prime}\left(\int_{t_{0}}^{t_{f}} K(\tau) u_{1}(\tau) d \tau-b\right)+ \\
\alpha \int_{t_{0}}^{t_{f}}\left(\dot{u}_{1}^{\prime}(\tau) P(\tau) \dot{u}_{1}(\tau)+u_{1}^{\prime}(\tau) Q(\tau) u_{1}(\tau)\right) d \tau
\end{gathered}
$$

where $\alpha>0$ is a regularization parameter, sign " ' " stands for transposition, and $P$ and $Q$ are positive definite weight matrices. Of course, here $\alpha$ should be significantly smaller than $\varepsilon$, for example, $\alpha=O\left(\varepsilon^{k}\right), k \geq 2$. Here, we will find the solution of problem (14) approximately, using an auxiliary nonlinear programming problem, for example, assuming that each coordinate in $u_{1}$ is represented using a series of orthogonal functions, so that

$$
u_{1, i}(\tau(t))=\frac{1}{2} c_{i, 0}+\sum_{j=1}^{k_{u}}\left(c_{i, 2 j-1} \cos (j \tau)+c_{i, 2 j} \sin (j \tau)\right), \quad i=1, \ldots, r
$$

where

$$
\tau=-\frac{2 \pi t}{t_{0}-t_{f}}+\frac{\pi\left(t_{0}+t_{f}\right)}{t_{0}-t_{f}}, \quad t \in\left[t_{0}, t_{f}\right], \quad \tau \in[-\pi, \pi],
$$

and $c_{l}$ are the vectors of unknown coefficients for each vector function $u_{1}(t)$ coordinate, $l=0,1, \ldots, 2 k_{u}$; the $k_{u} \geq 0$ is a parameter determines the number of terms in the series.

Thus, the coefficients $c_{i, l}(\alpha)$ are found using an iterative algorithm for solving the nonlinear programming problem

$$
M^{\alpha} \rightarrow \min _{c_{i, l}}
$$

where the next operations are made at each step $p$, so the scheme of algorithm have the next form:

(1) Values of $c_{i, l}^{(p+1)}$ are calculated by minimizing $M^{\alpha}\left(c_{i, l}^{(p)}, \alpha(p)\right)$;

(2) $\alpha^{(p+1)}=\alpha^{(p)} \gamma, \quad 0<\gamma<1$;

(3) If the specified accuracy of $M^{\alpha}$ is achieved, then stop.

Assuming that $u_{1, i}$ are found exactly, by the successive approximations approach, we can establish the following theorem under additional smoothness conditions for a function $F_{1}$.

Theorem 1. Let the vector function $F_{0}$ be such function that the system in (2) is flat and $u_{0}(t)$ is the open-loop control in problem (2) obtained by means of algorithm $[9,10]$, the regularization parameter $\alpha=O\left(\varepsilon^{k}\right)$, where $k \geq 2, F_{1}$ is twice differentiable of all variables, the coordinates $u_{1, i}$ are found exactly. Then, there is such a perturbation parameter $\varepsilon_{0}>0$ thatfor all $0<\varepsilon \leq \varepsilon_{0}$

1. $\left\|x\left(t_{f}, u_{0}\left(t_{f}\right)+\varepsilon u_{1}\left(t_{f}\right)\right)-x_{f}\right\|=\theta_{1}=o(\varepsilon)$,

2. $\left\|x\left(t_{f}, u_{0}\left(t_{f}\right)+\varepsilon u_{1}\left(t_{f}\right)\right)+\varepsilon \omega\left(t_{f}\right)-x_{f}\right\|=\theta_{2}<\theta_{1}$, if $\|\omega(t) \neq 0\|$ for all $t \in\left(t_{0}, t_{f}\right)$. 
The proof is carried out similarly to the scheme in [14] for smooth perturbations of the optimal control problem.

Note that during the practical use of the theorem, for greater accuracy of the final condition approximation, it is necessary to match the regularization parameter $\alpha$ to the accuracy of the solution of the regularized problem.

If a terminal control error is unacceptable for a given real-world problem, then auxiliary control $\omega(t)$ may be used to compensate for it. In that case, $\omega(t)$ is found as a solution in Problem 2, i.e., it minimizes

$$
e^{\prime} e \rightarrow \min _{d_{i, l}}
$$

where residual has the form

$$
e=x\left(t_{f}\right)-x\left(t_{f}, u_{0}(t)+\varepsilon\left(u_{1}(t)+\omega\left(\tau(t), d_{i, l}\right)\right)\right), \quad i=1, \ldots, r, \quad l=0, \ldots, k_{\omega},
$$

and controls $u_{0}$ and $u_{1}$ are already known.

Let us note that $\omega\left(\tau(t), d_{i, l}\right)$ is represented in the form of series (15), the number of members of which is determined by the parameter $k_{\omega} \geq 0$.

\section{Numerical Experiments}

Let us consider the problem

$$
\left.\begin{array}{l}
\dot{x}_{1}=u_{1}+\varepsilon\left(x_{3}+u_{2}\right), \quad x(0)=\left[\begin{array}{c}
5 \\
-2 \\
3
\end{array}\right]=\left[\begin{array}{l}
x_{0,1} \\
\dot{x}_{2}=x_{1}+x_{3} u_{1}, \\
\dot{x}_{3}=u_{2},
\end{array} x_{0,3}\right.
\end{array}\right] \quad x(2)=\left[\begin{array}{l}
0 \\
0 \\
0
\end{array}\right] .
$$

This problem has no practical meaning, but used to demonstrate the effectiveness of the proposed approach. The corresponding unperturbed dynamical system

$$
\begin{aligned}
& \dot{x}_{1}=u_{1}, \\
& \dot{x}_{2}=x_{1}+x_{3} u_{1}, \\
& \dot{x}_{3}=u_{2}
\end{aligned}
$$

is flat with the flat output

$$
y_{1}=x_{1}, \quad y_{2}=x_{2}
$$

Indeed, all state and control variables can be expressed in terms of flat output as follows:

$$
x_{1}=y_{1}, \quad x_{2}=y_{2}, \quad u_{1}=\dot{y}_{1}, \quad x_{3}=\frac{\dot{y}_{2}-y_{1}}{\dot{y}_{1}}, \quad u_{2}=\frac{\left(\ddot{y}_{2}-\dot{y}_{1}\right) \dot{y}_{1}-\left(\dot{y}_{2}-y_{1}\right) \ddot{y}_{1}}{\dot{y}_{1}^{2}} .
$$

It can be shown that the original system in (16) is not flat. For (16) we have

$$
\begin{array}{cc}
F_{0}=\left[\begin{array}{c}
u_{1} \\
x_{1}+x_{3} u_{1} \\
u_{2}
\end{array}\right], \quad F_{1}=\left[\begin{array}{c}
x_{3}+u_{2} \\
0 \\
0
\end{array}\right], \quad F_{0, x}\left(t, x_{0}(t), u_{0}(t)\right)=\left[\begin{array}{ccc}
0 & 0 & 0 \\
1 & 0 & u_{0,1} \\
0 & 0 & 0
\end{array}\right], \\
F_{0, u}\left(t, x_{0}(t), u_{0}(t)\right)=\left[\begin{array}{cc}
1 & 0 \\
x_{0,3} & 0 \\
0 & 1
\end{array}\right] .
\end{array}
$$


The matrix $\Phi$ for the homogeneous system corresponding to (12), the kernel matrix $K$, and the vector $b$ have the following representations

$$
\begin{gathered}
\Phi(t)=\left[\begin{array}{ccc}
1 & 0 & 0 \\
t & 1 & \int_{0}^{t} u_{0,1}(s) d s \\
0 & 0 & 1
\end{array}\right], \quad K\left(t, x_{0}(t), u_{0}(t)\right)=\left[\begin{array}{cc}
1 & 0 \\
-\tau+x_{0,3}(t) & -\left(\int_{0}^{t} u_{0,1}(s) d s\right) \\
0 & 1
\end{array}\right], \\
b\left(t, x_{0}(t), u_{0}(t)\right)=-\int_{0}^{T}\left[\begin{array}{c}
x_{0,3}(\tau)+u_{0,2}(\tau) \\
-\tau x_{0,3}(\tau)-\tau u_{0,2}(\tau) \\
0
\end{array}\right] d \tau .
\end{gathered}
$$

Following the algorithm of the covering method $[9,10]$, we introduce the additional state variable

$$
\xi=u_{1}=\dot{y}_{1} .
$$

The extended system

$$
\begin{aligned}
& \dot{x}_{1}=\xi, \\
& \dot{x}_{2}=x_{1}+x_{3} \xi, \\
& \dot{x}_{3}=\frac{1}{\xi}\left(\ddot{y}_{2}-\xi-x_{3} \ddot{y}_{1}\right), \\
& \dot{\xi}=\ddot{y}_{1}
\end{aligned}
$$

is equivalent to the normal Brunovsky form of the second order

$$
\ddot{y}_{1}=v_{1}, \quad \ddot{y}_{2}=v_{2},
$$

where $v_{1}, v_{2}$ stands for the new control input and $\xi$ represents the dynamic feedback. We extend the boundary problem (16) with the initial conditions on $\xi$ and rewrite the problem in terms of flat output as follows

$$
\begin{gathered}
y_{0,1}^{0}=y_{1}(0)=x_{0,1}^{0} \quad y_{0,2}^{0}=y_{2}(0)=x_{0,2}^{0} \quad \dot{y}_{0,1}^{0}=\dot{y}_{1}(0)=\xi_{0}, \\
y_{0,1}^{f}=y_{1}(2)=x_{0,1}^{f} \quad y_{0,2}^{f}=y_{2}(2)=x_{0,2}^{f} \quad \dot{y}_{0,1}^{f^{\prime}}=\dot{y}_{1}(2)=\xi_{f}, \\
\dot{y}_{0,2}^{0}=\dot{y}_{2}(0)=x_{0,1}^{0}+x_{0,3}^{0} \xi_{0}, \\
\dot{y}_{0,2}^{f}=\dot{y}_{2}(2)=x_{0,1}^{f}+x_{0,3}^{f} \xi_{f} .
\end{gathered}
$$

We suggest that we find the solution in the space of third-order polynomials

$$
y_{i}^{(4)}=0, \quad i=\{1,2\} .
$$

Let us consider the function

$$
p_{i}=y_{i}-\frac{1}{2}\left(t_{f}-t\right)^{2} \ddot{y}_{i}-\frac{1}{3}\left(t_{f}-t\right)^{3} \dddot{y}_{i}, \quad i=1,2 .
$$

The time derivatives of these functions are

$$
\begin{aligned}
& \dot{p}_{i}=\dot{y}_{i}+\left(t_{f}-t\right)^{2} \ddot{y}_{i}+\frac{1}{2}\left(t_{f}-t\right)^{3} \dddot{y}_{i}, \\
& \ddot{p}_{i}=0, \quad i=1,2 .
\end{aligned}
$$

From the initial conditions (22), we have

$$
p_{i}\left(t_{f}\right)=y_{0, i^{\prime}}^{f} \quad \dot{p}_{i}\left(t_{f}\right)=\dot{y}_{0, i^{\prime}}^{f} \quad i=\{1,2\} .
$$

The solution of Cauchy problem (25) and (26) in reverse time has the form

$$
p_{1}(t)=\xi_{f}\left(t-t_{f}\right)+x_{0,1}^{f}, \quad p_{2}(t)=\left(x_{0,1}^{f}+x_{0,3}^{f} \xi_{f}\right)\left(t-t_{f}\right)+x_{0,2}^{f} .
$$


Substituting $t=t_{0}$ in these formulas we get the initial conditions on $p_{i}$ and $\dot{p}_{i}$ which allows us to find the initial conditions on $\ddot{y}_{i}$ and $\dddot{y}_{i}$, using (24) and (25). With (22) we have the full set of initial conditions for (23). Solving the Cauchy problem (23) with the initial conditions we can receive the solution of point-to-point problem in terms of flat output. Then, (19) give us the explicit representations for the coordinates of admissible trajectories and the corresponding controls. For brevity, we give the control representations only

$$
\begin{aligned}
& u_{0,1}(t)=-\frac{1}{T^{3}}\left(-\xi_{0}\left(t-t_{f}\right)\left(3 t-2 t_{0}-t_{f}\right) T+\left(t-t_{0}\right)\left(-\xi_{f}\left(3 t-t_{0}-2 t_{f}\right) T-\right.\right. \\
& \left.-6\left(t-t_{f}\right)\left(x_{0,1}^{0}-x_{0,1}^{f}\right)\right) \\
& u_{0,2}(t)=\frac{1}{m_{1}^{2}}\left(m _ { 1 } \left(2\left(-t_{f}+\Delta t_{3}\right) m_{2}+\left(8 x_{0,1}^{f}+4 x_{0,1}^{0}\right) \Delta t_{3} t_{f}+\right.\right. \\
& \left.+m_{7}-12 t m_{4}+2 t_{f} m_{3}+\left(-8 x_{0,1}^{f}-4 x_{0,1}^{0}\right) \Delta t_{3} t_{f}+\xi_{0} T m_{5}+\xi_{f} T m_{6}\right)- \\
& \left.-2 m_{8}\left(\xi_{0} \Delta t_{2}\left(2 t_{f}-\Delta t_{3}\right) m_{9}-\Delta t_{1}\left(-2 \Delta t_{2} m_{10}-m_{11}\right)\right)\right)
\end{aligned}
$$

where $\xi_{0}=-8, \xi_{f}=-4$ and

$$
\begin{aligned}
& T=t_{f}-t_{0}, \quad n_{1}=3 t-2 t_{0}-t_{f}, \quad n_{2}=2 t_{0}+t_{f}+3 x_{0,3}^{f}, \\
& n_{3}=3 t-t_{0}-2 t_{f}, \quad n_{4}=x_{0,1}^{0}-x_{0,1}^{f}, \\
& \Delta t_{1}=t-t_{0}, \quad \Delta t_{2}=t-t_{f}, \quad \Delta t_{3}=t_{0}+t_{f}, \\
& m_{1}=-\xi_{0} \Delta t_{2} n_{1} T+\Delta t_{1}\left(-\xi_{f} n_{3} T-6 \Delta t_{2} n_{4}\right), \\
& m_{2}=\left(-2 x_{0,1}^{f}+2 x_{0,1}^{0}\right) t_{f}-3 x_{0,2}^{f}+3 x_{0,2}^{0} \\
& m_{3}=\left(x_{0,1}^{f}+2 x_{0,1}^{0}\right) t_{f}-3 x_{0,2}^{f}+3 x_{0,2}^{0}{ }^{\prime} \\
& m_{4}=\left(x_{0,1}^{f}+x_{0,1}^{0}\right) t_{f}-\Delta t_{3} x_{0,1}^{f}-x_{0,2}^{f}+x_{0,2}^{0}, \\
& m_{5}=2\left(-t_{f}+\Delta t_{3}\right)\left(t_{f}+x_{0,3}^{0}\right)-2 t\left(t_{f}+\Delta t_{3}+3 x_{0,3}^{0}\right)+t_{f}\left(t_{f}+4 x_{0,3}^{0}\right)+3 t^{2}, \\
& m_{6}=2\left(-t_{f}+\Delta t_{3}\right)\left(t_{f}+2 x_{0,3}^{f}\right)+t_{f}^{2}+\left(2 x_{0,3}^{f}-2 \Delta t_{3}\right) t_{f}+\Delta t_{3}-2 t n_{2}+3 t^{2}, \\
& m_{7}=\left(-2 x_{0,1}^{0}-4 x_{0,1}^{f}\right) t_{f}^{2}+\left(8 \Delta t_{3} x_{0,1}^{f}+4 \Delta t_{3} x_{0,1}^{0}\right) t_{f}-2\left(x_{0,1}^{0}+2 x_{0,1}^{f}\right) \Delta t_{3}^{2}+6 t^{2} n_{4}, \\
& m_{8}=3 n_{4}\left(3 t-\Delta t_{3}\right)-\xi_{f} n_{1} T-\xi_{0} n_{3} T, \\
& m_{9}=\left(-t_{f}+\Delta t_{3}\right)\left(t_{f}+2 x_{0,3}^{0}\right)-t\left(\Delta t_{3}+3 x_{0,3}^{0}\right)+t_{f} x_{0,3}^{0}+t^{2}, \\
& m_{10}=3 x_{0,2}^{f}-3 x_{0,2}^{0}-3\left(x_{0,1}^{0}+x_{0,1}^{f}\right) t_{f}+t n_{4}+\Delta t_{3}\left(x_{0,1}^{0}+2 x_{0,1}^{f}\right), \\
& m_{11}=\xi \xi_{f} T\left(\left(-t_{f}+\Delta t_{3}\right)\left(t_{f}+x_{0,3}^{f}\right)-t\left(\Delta t_{3}+3 x_{0,3}^{f}\right)+2 t_{f} x_{0,3}^{f}+t^{2}\right), \\
& x_{0,1}(0)=x_{0,1}^{0} \quad x_{0,2}(0)=x_{0,2}^{0} \quad x_{0,3}(0)=x_{0,3}^{0} \\
& x_{0,1}(2)=x_{0,1}^{f} \quad x_{0,2}(2)=x_{0,2}^{f} \quad x_{0,3}(2)=x_{0,3}^{f} .
\end{aligned}
$$

The Figure 1a shows the results of numerical simulation of closed-loop systems along $u_{0}(t)$ and $u_{1}(t, \varepsilon)=u_{0}(t)+\varepsilon u_{1}(t)$ for $\varepsilon=0.1, k_{u}=2$. The initial approximations $\alpha^{(0)}=1$, $c_{i, l}^{(0)}=0$ were used for the $u_{1}(t)$ calculation. Achievement of a given calculation accuracy for $M^{\alpha}$ occurs in 14 iterations and does not depend on the parameter $\varepsilon$. The Figure $1 \mathrm{~b}$ shows the trajectories of systems for $\varepsilon=1$ along four controls, namely, $u_{0}(t), u_{1}(t, \varepsilon)$ and $u_{\omega}(t, \varepsilon)=u_{1}(t, \varepsilon)+\varepsilon \omega(t)$, here the correction $\omega$ was calculated for $k_{\omega}=1$, and $\hat{u}(t)$. The last control is found by reducing the problem (16) to a nonlinear programming problem

$$
\left(x\left(t_{f}\right)-x\left(t_{f}, \hat{u}\right)\right)^{\prime}\left(x\left(t_{f}\right)-x\left(t_{f}, \hat{u}\right)\right) \rightarrow \min _{\hat{u}}
$$

where $\hat{u}$ is searched as a series (15). We found $\hat{u}(t)$ to compare with the proposed controls $u_{0}(t), u_{1}(t, \varepsilon)$ and $u_{\omega}(t, \varepsilon)$. Let us note that to achieve the same solution accuracy that was obtained for $u_{\omega}(t, \varepsilon)$, the number of unknown coefficients of the series (15) in the experiments turned out to be 7 , i.e., the parameter $k_{\hat{u}(t)}$ in (15) was equal to 3 . 

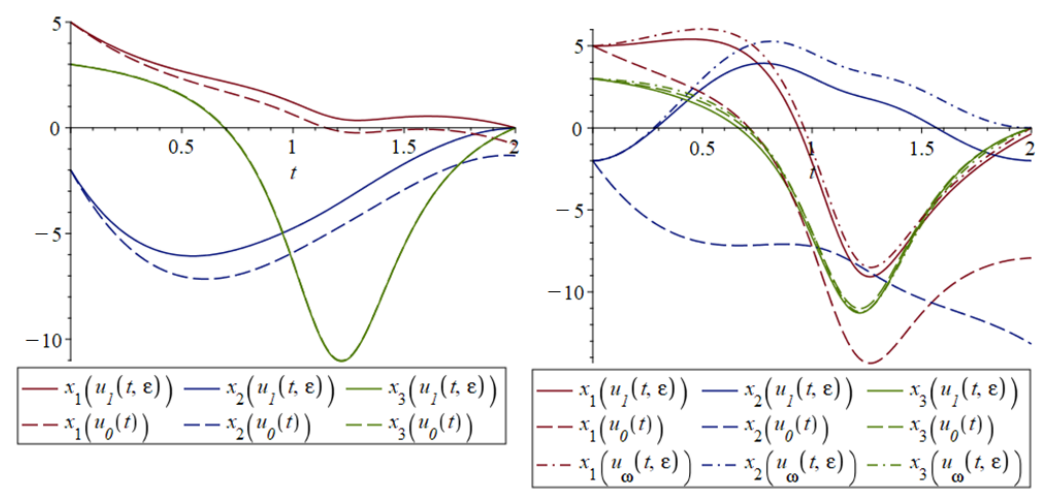

Figure 1. (left) Trajectories of the closed-loop systems for $\varepsilon=0.1$. (right) Trajectories of the closedloop systems for $\varepsilon=1$.

Table 1 presents the numerical experiments results for various $\varepsilon$, where $J(v)=$ $x\left(t_{f}, v\right)^{\prime} x\left(t_{f}, v\right), T(v)$ denotes the time in seconds spent to search for control $v$. The calculation $T\left(u_{\omega}\right)$ also took into account the time needed for $u_{1}(t, \varepsilon)$ calculation. To clarify the role of correction $\varepsilon u_{1}(t)$ in the search process of $\omega(t)$, Table 1 also provides the results for the control $u_{0}(t)+\varepsilon \omega(t)$ in which this correction did not present.

Table 1. Numerical experiments results for various $\varepsilon$.

\begin{tabular}{ccccccc}
\hline$\varepsilon$ & $J\left(u_{0}(t)\right)$ & $J\left(u_{\mathbf{1}}(t, \varepsilon)\right)$ & $J\left(u_{\omega}(t, \varepsilon)\right)$ & $T\left(u_{\omega}(t, \varepsilon)\right)$ & $T\left(u_{0}(t)+\varepsilon \omega(t)\right)$ & $T(\hat{u}(t))$ \\
\hline 1 & 15.374 & 2.082 & $0.12 \cdot 10^{-3}$ & 1.909 & 2.524 & 2.963 \\
0.5 & 7.678 & 0.507 & $0.12 \cdot 10^{-3}$ & 1.872 & 1.994 & 2.911 \\
0.1 & 1.537 & 0.023 & $0.12 \cdot 10^{-3}$ & 1.242 & 1.645 & 3.460 \\
0.01 & 0.154 & $0.20 \cdot 10^{-3}$ & $0.11 \cdot 10^{-3}$ & 0.814 & 1.313 & 3.083 \\
0.001 & 0.015 & $3.22 \cdot 10^{-6}$ & $3.22 \cdot 10^{-6}$ & 0.587 & 0.969 & 3.046 \\
\hline
\end{tabular}

As one may see from Table 1, the time for calculating the correction of the point-topoint control for reaching the end point with a given accuracy decreases with an increase in the order of asymptotic approximations, i.e., $T(\hat{u}(t))>T\left(u_{0}(t)+\varepsilon \omega(t)\right)>T\left(u_{\omega}(t, \varepsilon)\right)$, and the direct search for the terminal control $\hat{u}(t)$ takes considerably longer.

Note that the time of $\hat{u}(t)$ calculation for different values of the parameter $\varepsilon$ is approximately the same, and the calculation time of $T\left(u_{\omega}(t, \varepsilon)\right)$ decreases with decreasing $\varepsilon$. The last is natural, as with a decrease in this parameter, the asymptotics more accurately approximates the exact solution and, as a consequence, the terminal error decreases, which can be seen from the comparison of the first two columns in Table 1.

\section{Conclusions}

An approach to the construction of control in a nonlinear perturbed point-to-point problem is proposed. It consists in correction of admissible control in the unperturbed problem obtained from the Fredholm integral equation of the first kind. To solve that problem, Tikhonov's regularizing smoothing functional is used. A statement of terminal error along the found control is formulated. A method for further refinement of control using an auxiliary variational problem is proposed. The conducted numerical experiments demonstrate the effectiveness of the theoretical results obtained. The obtained theoretical results may be used to computationally efficient design of terminal control in various applied problems.

Author Contributions: Conceptualization, M.D.; methodology, M.D.; formal analysis, Y.B. and D.M.; investigation, Y.B. and D.M.; writing—original draft preparation, Y.B., M.D. and D.M.; writingreview and editing, Y.B., M.D. and D.M.; visualization, D.M.; supervision, M.D. All authors have read and agreed to the published version of the manuscript. 
Funding: The research was funded by RFBR, projects numbers 18-37-20032 and 20-57-00011.

Institutional Review Board Statement: Not applicable.

Informed Consent Statement: Not applicable.

Data Availability Statement: The data presented in this study are available on request from the corresponding author.

Conflicts of Interest: The authors declare no conflict of interest.

\section{References}

1. Dmitriev, M.G.; Makarov, D.A. A design of an approximate regulator for a weakly nonlinear terminal control problem using the penalty functions method. AIP Conf. Proc. 2018, 1997. [CrossRef]

2. Krischenko, A.P.; Kanatnikov, A.N.; Tkachev, S.B. Plannind and Control of Spatial Motion of Flying Vehicles. In Proceedings of the IFAC Workshop Aerospace Guidance, Navigation and Flight Control Systems AGNFCS'09, Samara, Russia, 30 June-2 July 2009. Available online: http:/ /lib.physcon.ru/doc?id=b502c4579298 (accessed on 11 June 2021).

3. Krishchenko, A.P.; Fetisov, D.A. Terminal control problem for affine systems. Differ. Equ. 2013. 49, 1378-1388. [CrossRef]

4. Fetisov D.A. Reshenie terminal'nykh zadach dlya mnogomernykh affinnykh sistem na osnove preobrazovaniya k kvazikanonicheskomu vidu. Vestnik MGTU im. Baumana. Estestvennye Nauki 2014, 5, 16-31. (In Russian)

5. Fliess, M.; Levine, J.; Martin, P.; Rouchon, P. A Lie-Bäcklund Approach to Equivalence and Flatness of Nonlinear Systems. IEEE Trans. Autom. Control. 1999, 44, 922-937. [CrossRef]

6. Belinskaya, Y.S.; Chetverikov, V.N. Covering Method for Terminal Control with Regard of Constraints. Differ. Equ. 2014, 50, 1632-1642. [CrossRef]

7. Sira-Ramirez, H.; Castro-Linares, R.; Liceaga-Castro, E. A Liouvillian Systems Approach for the Trajectory Planning-based Control of Helicopter Models. Int. J. Robust Nonlinear Control. 2000, 10, 301-320. [CrossRef]

8. Chetverikov, V.N. Liouville Systems and Symmetries. Differ. Equ. 2012, 48, 1639-1651. [CrossRef]

9. Chetverikov, V.N. Flat control systems and deformations of structures on diffieties. Forum Math 2004, 16, 903-923. [CrossRef]

10. Belinskaya, Y.S.; Chetverikov, V.N. Covering Method for Point-to-Point Control of Constrained Flat System. IFAC-Papers OnLine 2015, 48, 924-929. [CrossRef]

11. Gornov, A.Y.; Dmitriev, M.G.; Tyatyushkin, A.I. Experience in Solving Optimal Control Problems with A Boundary Layer (Opyt Resheniya Zadach Optimal'Nogo Upravleniya S Pogranichnym Sloem); VINITI DEP: Moscow, Russia, 1985; No. 8441-V85; 19p. (In Russian)

12. Tikhonov, A.N.; Arsenin, V.Y. Methods for Solving Incorrect Problems (Metody Resheniya Nekorrektnyh Zadach); Publishing House "Nauka": Moscow, Russia, 1979; 284p. (In Russian)

13. Vasil'eva, A.B.; Tikhonov, N.A. Integral Equations (Integral'Nye Uravneniya); Fizmatlit: Moscow, Russia, 2004; 160p. (In Russian)

14. Gnevko, S.V.; Kalinin, A.I. Asymptotic optimization of nonlinear regularly perturbed control systems. USSR Comput. Math. Math. Phys. 1991, 31, 28-37. 